\title{
Criminologie
}

\section{Recherche sur la violence familiale : contribution des différentes épistémologies}

\section{Carmen Poulin et Lynda R. Ross}

Volume 30, numéro 2, automne 1997

Violences familiales

URI : https://id.erudit.org/iderudit/017402ar

DOI : https://doi.org/10.7202/017402ar

Aller au sommaire du numéro

\section{Éditeur(s)}

Les Presses de l'Université de Montréal

ISSN

0316-0041 (imprimé)

1492-1367 (numérique)

Découvrir la revue

Citer cet article

Poulin, C. \& Ross, L. R. (1997). Recherche sur la violence familiale : contribution des différentes épistémologies. Criminologie, 30(2), 7-25.

https://doi.org/10.7202/017402ar
Résumé de l'article

Knowledge, over the last century, has been grounded mainly in the institution of empirical science. This epistemological tradition is tightly linked to positivism and objectivity. Feminists from various disciplines, including that of Criminology, have become disillusioned in the ability of traditional empiricism to produce knowledge that is relevant, historically and socially, to women, and based in their everyday experience. Feminists have proposed alternative epistemological frameworks to explore questions driven by political feminist agendas. In the present article, an overview of these new epistemological frameworks is presented to develop an evaluation grid. Using this grid, studies from different traditions in the area of family violence and violence against women are examined and critiqued to determine how each epistemological framework can advance (or not) the feminist agenda. 
RECHERCHE SUR LA VIOLENCE FAMILIALE : CONTRIBUTION DES DIFFÉRENTES ÉPISTÉMOLOGIES

Carmen Poulin'

Lynda R. Ross ${ }^{2.3}$

Knowledge, over the last century, has been grounded mainly in the institution of empirical science. This epistemological tradition is tightly linked to positivism and objectivity. Feminists from various disciplines, including that of Criminology, have become disillusioned in the ability of traditional empiricism to produce knowledge that is relevant, historically and socially, to women, and based in their everyday experience. Feminists have proposed alternative epistemological frameworks to explore questions driven by political feminist agendas. In the present article, an overview of these new epistemological frameworks is presented to develop an evaluation grid. Using this grid, studies from different traditions in the area of family violence and violence against women are examined and critiqued to determine how each epistemological framework can advance (or not) the feminist agenda.

Les théoriciennes féministes travaillant sur les rapports des femmes au pénal doivent s'investir dans des objets beaucoup plus larges, par exemple la critique féministe des connaissances et des disciplines particulières : celle-ci est déjà très bien amorcée mais plus fondamentalement, c'est la critique de la science et la reconstruction historique des disciplines qu'il faut faire; il faut aussi entreprendre ou poursuivre la critique féministe de l'éthique et de la normativité. (Bertrand, 1994, p. 62)

Un questionnement en ce qui touche le rôle de «l'institution de Ia science » est en pleine expansion dans de nombreuses disciplines, y compris

1. Professeure de psychologie, Université du Nouveau-Brunswick, B.P. \# 45444, Frédéricton, N.-B., E3B 6E4. Adresse électronique : carmen@unb.ca

2. Département de psychologie, Université du Nouveau-Brunswick, B.P. \# 45444, Frédéricton, N.-B., E3B 6E4. Adresse électronique : y20g@jupiter.sun.csd.unb.ca

3. Les auteures tiennent à exprimer leur gratitude à l'endroit de Pierre Petit, Yvonne Poulin et deux évaluateur/trice/s anonymes pour leur lecture d'une première version de ce texte et pour les commentaires pertinents qu'ils ont formulés à son endroit. 
la criminologie et ses disciplines connexes telles la sociologie et la psychologie. Pour les chercheur/euse/s œuvrant dans ces disciplines, les connaissances qui ont été acquises, ainsi que les croyances, les attentes et la compréhension de la " réalité ", de la « vérité » proviennent largement de cette institution qu'est la science. Mais l'omission, la fausse représentation historique et chronique des femmes et de leurs préoccupations dans le contexte du savoir (dit) scientifique a amené un grand nombre de féministes à mettre en question et à examiner, de façon critique, tous les aspects du processus scientifique.

Certain/e/s auteur/e/s ont utilisé l'expression « méthodes féministes de recherche » en se référant à des méthodes de recherche dites exclusivement féministes. Toutefois, en accord avec Harding (1989), nous croyons qu'il n'y a pas de méthode de recherche exclusivement féministe. Il y a plutôt des féministes cuvrant dans différentes disciplines, et utilisant une variété de méthodes de recherche pour mener des recherches féministes. Reinharz (1992) fait le point en exprimant l'opinion que le féminisme offre la perspective alors que la discipline offre la méthode. Ainsi, la distinction se trouve entre chercheur/euse/s féministes et chercheur/euse/s non féministes, plutôt qu'entre méthodes de recherche féministe et non féministe. Cela dit, nous voulons attirer l'attention sur le choix des méthodes qui « découle » du cadre épistémologique du/de la chercheur/euse, ainsi que sur le rôle de l'orientation politique de ce/cette dernier/ière. C'est l'interaction entre tous ces aspects que nous désirons explorer dans cette présentation, ainsi que le langage adopté pour décrire cette interaction.

Aucun secteur de recherche, surtout dans le domaine des sciences sociales, ne devrait échapper à cet examen, à ce questionnement. Nonobstant, dans cet article, nous avons choisi d'explorer cette dynamique à partir de l'abus et de la violence faite aux femmes. En particulier, nous examinons l'impact que peut avoir le choix épistémologique du/de la chercheur/euse, non seulement au niveau de la méthode de recherche adoptée, mais aussi en ce qui a trait aux résultats, à la présentation, l'explication, la compréhension et la «construction sociale » de la problématique étudiée. Nous avons tenté d'éclairer cette dynamique en employant deux stratégies : la « simplification » et l' « exemplification ». Il est intéressant de noter que ces deux stratégies se portent plus facilement à l'examen des épistémologies fondées sur la pensée positiviste que les épistémologies alternatives tel le postmodernisme. Étant psychologues de formation, nos choix de stratégies sont certes dus à cette (dé)formation. Toutefois, cette limitation informe par sa présence ; elle montre jusqu'à quel point le cadre épistémologique (dans ce cas, celui fondé sur la pensée positiviste) s'infiltre dans nos outils de travail. 
Notre présentation est divisée en trois parties. Dans la première, nous résumons le contenu de l'agenda politique féministe ${ }^{4}$ et son influence sur la recherche. En deuxième lieu, nous introduisons brièvement différents cadres épistémologiques développés par les théoriciennes féministes. En guise d'introduction à cette même section, nous discutons la notion de l'objectivité en recherche. Dans la troisième section, nous examinons, à titre d'exemple, quelques études sur la violence faite aux femmes qui ont été conduites à partir de différents cadres épistémologiques.

\section{L'AGENDA POLITIQUE FÉMINISTE : UN GUIDE DE RECHERCHE}

L'interaction entre les différents objectifs politiques féministes, cadres épistémologiques et choix de méthodes de recherche représente une dynamique complexe avec laquelle le/la chercheur/euse féministe doit composer. Évidemment, les positions politiques féministes ne sont pas toutes les mêmes. Néanmoins, il est possible d'identifier certains points communs. Tout féminisme suggère que les femmes ont été systématiquement et «systémiquement » exploitées, opprimées et dévaluées. Cela dit, à partir de ses résultats, la recherche féministe a pour but de contribuer à l'élimination des conditions qui causent cette situation pour les femmes (Allen et Barber, 1992). À la base de l'agenda politique féministe, on trouve donc la nécessité de déséquilibrer, d'abolir ou de modifier les sources de pouvoirs discriminatoires basées sur le genre ${ }^{5}$ et le sexe (Kremer, 1990). Par conséquent, dans la mesure où cet agenda sert de guide pour la recherche féministe, les femmes devront se retrouver au centre de cette recherche et profiter de ses résultats. Par « profit », nous entendons surtout l'émancipation des femmes et l'amélioration de leurs conditions de vie (Thompson, 1992). De plus, Harding (1987) insiste sur le fait qu'un des objectifs de la recherche féministe doit être de neutraliser les forces qui ont exclu, subordonné et rendu victimes les femmes historiquement. Toute recherche féministe se veut libératrice, en donnant un juste pouvoir aux femmes et en aidant à transformer le système

4. Comme nous en discuterons plus loin, il est évident qu'il existe plus d'un agenda féministe. Toutefois, certains postulats sont à la base de tous ces agendas férninistes. Par exemple, les féministes s'entendent pour dire que les femmes ont été exploitées, opprimées, et dévaluées et que le féminisme a pour objectif d'augmenter le niveau de conscience a l'egard de l'inégalité entre les hommes et les femmes. Par conséquent, nous utilisons l'expression « agenda féministe " pour désigner l'ensemble de ces postulats communs.

5. Par " genre ", on entend " la construction sociale » de ce qu'est le sexe d'une personne, ou la " nature » de la femme ou de l'homme. Chez l'individu, le genre se développe à travers ses interactions dans un contexte social et « façonné ». De notre compréhension de ce concept découle notre conceptualisation de ce qu'est la féminité, la masculinité, et tout un ordre de relations sociales guidées par cette catégorisation. 
patriarcal (Cook et Fonow, 1990). Étant donné cet objectif politique qui guide la recherche féministe, l'illusion d'objectivité ne peut pas et ne doit même pas exister.

Il existe différentes façons à partir desquelles l'agenda politique féministe peut orienter la recherche féministe dans ses objectifs. En général, il semble que cette recherche soit motivée par une prise de conscience des conséquences liées à la position des femmes dans le contexte patriarcal. Par conséquent, le but de la recherche féministe doit inclure la documentation, l'explication, la déconstruction et, finalement, l'élimination du patriarcat comme structure sociale.

\section{SURVOL DE DIFFÉRENTS CADRES ÉPISTÉMOLOGIQUES}

Pour aborder notre discussion sur les différents cadres épistémologiques, il faut avant tout répondre à deux questions fondamentales afin de comprendre pourquoi les féministes ont développé des approches alternatives à l'empirisme traditionnel. Ces deux questions sont les suivantes : 1) qu'est-ce qui a précipité la recherche de nouvelles épistémologies ? et 2) pourquoi changer la notion d'objectivité ?

\section{Qu'est-ce qui a précipité la recherche d'autres épistémologies ?}

Comme nous l'avons mentionné précédemment, le manque de représentation des femmes et de leurs préoccupations dans le domaine des sciences sociales ${ }^{6}$ a mené les chercheur/euse/s féministes au désillusionnement en regard de l'institution de la science, de l'empirisme traditionnel et, encore plus fondamentalement pour certain/e/s, du positivisme. Comme le résume Bertrand (1993) :

They demonstrated that the facts were not all there yet were reported as complete, that the conclusions were often invalid because of the inferences of generalizability. They saw that the interpretations of the facts were presented as objective, neutral and as "the truth" while in fact they were not only subjective, which is always the case, but biased and often sexist. (p. 2)

En effet, ce qui fait partie du savoir au sujet du monde en général n'est, en réalité, qu'une réflexion de ce qui est connu au sujet des hommes ; ce qui fait partie du savoir au sujet des femmes est, forcément, une distorsion de leurs réalités (Tavris, 1992 ; Weisstein, 1972).

6. La critique de l'institution de la science n'est pas limitée aux sciences sociales. Voir, par exemple, les travaux d'Adeline Fox Keeler (1985) et Roberta Mura (1991). 
Un cadre épistémologique représente une théorie du savoir, une position philosophique face à nos connaissances et à notre façon de les acquérir (Crawford et Marecek, 1989). De plus, le cadre épistémologique sert de stratégie justificative pour la recherche de la " vérité », la « réalité » et l'« objectivité ». Toutefois, les épistémologies développées par les féministes constituent un défi au niveau de l'éthique, de la normativité, de la politique et des postulats fondamentaux de "l'institution de la création du savoir " (Bertrand, 1994; Harding, 1991). Les féministes ne sont pas les seul/e/s à avoir lancé ce défi. Cependant, elles se sont engagées dans ce projet car, comme le dit Harding (1990), « [... they] need a defence against, and an alternative, positive program to, the traditional discourses of both objectivism and interpretationism $»($ p. 87).

Dans le contexte du courant de pensée positiviste, ainsi que dans l'épistémologie de l'empirisme traditionnel qui en découle, il est possible de contrôler et de prédire (Lather, 1991). Un des postulats sur lesquels est basé l'empirisme traditionnel (et même la pensée positiviste), est que les sens offrent l'accès à la " vérité » et à sa vérification. En d'autres mots, la nature, l'environnement, les comportements peuvent être décrits, compris et interprétés de façon absolue sans que le savoir ou l'expérience de la personne qui observe soient pris en considération dans le processus de la recherche. Un autre postulat qui fonde l'empirisme traditionnel, c'est que rien ne se produit par hasard, tout est relié : chaque fait a sa cause. Ces postulats justifient et donnent du poids aux connaissances développées dans ce contexte épistémologique (Nielsen, 1990).

En fait, c'est l'abandon de la présomption d'un aspect important de ces postulats - c'est-à-dire l'objectivité de l'observateur/trice ou chercheur/euse - qui a conduit les féministes au scepticisme. Leur critique porte sur tous les aspects de la recherche, de la formulation du problème à la diffusion des résultats. Comme le suggèrent Allen et Barber (1992), la critique féministe de la pensée positiviste est fondée sur la prise de conscience que la réalité sociale n'est pas, et n'a jamais été sans contexte. Lather (1991) ajoute que, de façon historique, l'observation empirique fut saturée par les influences personnelles et sociales qui ont biaisé l'observateur/trice. Selon cette dernière :

[...] power is assumed to permeate all aspects of our efforts to know and language is theorized as constitutive rather than representational [...] all researchers construct their object of inquiry out of the materials their culture provides, and values play a central role in this linguistically, ideologically and historically embedded project that we call science [...] (p. 104-105) 
Certaines théoriciennes ajoutent que la recherche empirique traditionnelle reproduit les relations du système patriarcal (Landrine et al., 1992). Par exemple, la relation de pouvoir qui existe entre « chercheur/euse/s expert/e/s » et « participant/e/s, objet de recherche ». Les participant/e/s sont érodé/e/s de leur expérience, de leur vécu ; les comportements sont détachés de leurs contextes sociaux.

\section{Pourquoi changer la notion d'objectivité ?}

La critique féministe du concept d'objectivité conduit à deux options : 1) rejeter complètement la notion d'objectivité et, par conséquent, la recherche empirique, ou 2) réviser cette notion afin qu'elle reflète ce qu'est vraiment l'objectivité. Harding (1987) suggère que si on abandonne complètement la notion d'objectivité, un glissement s'opère vers la pensée et le jugement relativistes. Afin d'éviter cette situation indésirable où n'existe plus aucun standard d'évaluation, d'acceptation ou de rejet pour l'examen de théories développées par des groupes utilisant différentes méthodes, la notion d'objectivité doit être élargie pour inclure la subjectivité, et accepter que la voix du/de la chercheur/cheuse soit présente à toutes les étapes de la recherche. Selon Harding, en reconnaissant que l'intérêt, les valeurs et la passion font partie de la recherche, l'objectivité est non seulement conservée, mais de plus, elle est renforcée. Redéfinir la notion d'objectivité nécessite un examen réfléchi des relations qui existent entre questions de recherche, chercheur/ euse/s et sujets/participant/e/s, plutôt que de nier l'existence de ces relations.

La recherche d'autres façons d'acquérir des connaissances, la redéfinition de la notion d'objectivité ont produit des cadres épistémologiques féministes représentant des alternatives au cadre empirique traditionnel. Trois épistémologies féministes ont émergé, permettant de nouveaux encadrements de recherche : l'empirisme féministe, le standpointisme et le féminisme postmoderne. Ces nouvelles épistémologies ont été développées non seulement en réaction au manque de connaissances, mais aussi en réponse à la nécessité de disposer de théories féministes pour guider la recherche.

\section{Épistémologies féministes}

L'empirisme féministe reste encore proche de ce qui est défini et accepté comme « la » méthode scientifique. C'est une approche conservatrice qui, jusqu'à présent, offre « [...] the most effective grounds for defending controversial claims about race, class, cultural differences » (Harding, 1990, p. 92). Les féministes « empiristes » insistent pour dire que les résultats sexistes et « androcentriques » sont simplement le reflet de mauvaises recherches ; pour elles/eux, il suffit de suivre les règles et principes de la science avec plus de précision et d'ardeur (Harding, 1991). Les recherches sont motivées 
par l'agenda politique féministe et, selon l'empirisme féministe, les préjugés peuvent être éliminés par des stratégies de contrôle de la méthode scientifique. Toutefois, identifier et contrôler des préjugés culturels requiert un complément de sensibilité que la méthode parvient difficilement à offrir.

L'avantage principal de l'empirisme féministe, c'est sa perspective « conservatrice » (Harding, 1991). Étant donné ses postulats, les affirmations générées à partir de la méthode scientifique dans les sciences humaines sont données pour « vraies ». Comme le suggère Lather (1991), la science représente « [...] one among many truth games » (p. 104). D'autre part, l'approche conservatrice féministe empirique permet d'explorer des questions qui restent invisibles dans un contexte qui nie l'existence de biais et de préjugés. Parlant dans un langage qui mime celui de l'empirisme traditionnel, les féministes empiristes augmentent alors leur chance d'être entendu/e/s. L'empirisme féministe a cependant été critiqué à son tour pour sa perspective qui « [...] embodies the intellectual and political sins of the Enlightenment » (Harding, 1990 , p. 84). Cette critique est venue plus particulièrement des féministes postmodernes (Di Stefano, 1990 ; Flax, 1990).

Le standpointisme se sépare de façon importante de l'empirisme traditionnel et même de la version féministe de l'empirisme, tout en gardant néanmoins l' « essence » de l'approche empirique. Le standpointisme découle de la pensée marxiste. Plus particulièrement, cette épistémologie s'inspire de l'interprétation marxiste de la relation entre l'oppresseur et l'opprimé/e, fondée sur la possibilité que l'opprimé/e possède une vision plus juste de la réalité, moins partiale, moins perverse que celle de l'oppresseur (Hartsock, 1987 a ; Smith, 1987). Tandis que la théorie marxiste est basée sur la relation entre la bourgeoisie et le prolétariat, le standpointisme met l'accent sur la relation entre les hommes comme oppresseurs, et les femmes en tant qu'opprimées. Selon le standpointisme, il y a peu d'intérêt pour les femmes à défendre la construction sociale actuelle de la féminité, en comparaison avec l'intérêt des hommes à défendre la construction sociale actuelle de la masculinité. Étant moins investies dans le statu quo, celles-ci peuvent théoriquement s'en distancer plus facilement. Cela dit, les femmes peuvent s'ouvrir à une conception élargie de la réalité et, ce faisant, mieux s'approcher de l'objectivité (Harding, 1991). Cette distance est essentielle à la production d'analyses innovatrices et critiques. C'est en effet à partir de cette distance que s'offrent de nouveaux points de vue (par exemple, une perspective qui se situe dans le contexte de la lutte historique des femmes contre la domination des hommes). De façon générale, les analyses sont venues de la perspective dominante, c'est-à-dire celle de l'homme et, pour Smith (1987), la perspective des femmes est différente, fondée sur le quotidien, la vie de tous les jours, c'est-à-dire une réalité concrète. 
Women's work processes children, food, all bodies, balky machines, and social relations. It makes possible men's retreat to an appropriation of 'abstract masculinity'. (Harding, 1991, p. 131)

Bref, le standpointisme réclame que l'expérience des femmes soit le point de départ pour le développement d'un savoir plus complet et moins déformé que celui élaboré à partir de l'expérience des hommes (Harding, 1990 ; Smith, 1987). Ce qu'il faut retenir du standpointisme, c'est que cette épistémologie est basée sur la reconnaissance qu' un point de vue est plus authentique, plus proche de la "vérité », s'il provient d'un groupe ayant moins de pouvoir social.

Les théoricien/ne/s standpointistes suggèrent que la majorité des problèmes inhérents à l'approche empirique nécessitent, pour faire avancer la science, bien plus que des corrections mineures. Le standpointisme, selon Harding (1991), attire ceux/celles qui comprennent et sont intéressé/e/s par la relation entre les « [...] patterns of thought and the historical conditions that make such patterns reasonable » (p. 134). S'intéressent aussi à cette approche ceux/celles qui acceptent que la réalité des femmes puisse représenter le point de départ pour le développement de connaissances plus complètes et moins erronées (Harding, 1991 ; Hartsock, 1987b ; Smith, 1987). Le standpointisme commence par s'attaquer aux bases de l'empirisme en suggérant que la réalité n'est pas qu'une structure ; l'ordre social présente au contraire plusieurs structures qui se croisent et entre lesquelles existent frictions et conflits (Harding, 1990).

Dans sa critique du standpointisme, Flax (1990) suggère que cette perspective est encore trop ancrée dans de faux postulats issus du siècle des lumières. Elle attire l'attention sur le fait qu' « avoir accès à la vérité » (une vérité plus vraie que la précédente) est une assertion basée sur des postulats problématiques, par exemple, le fait de croire qu'il soit possible pour les gens d'agir rationnellement selon leurs propres intérêts et que les femmes (ies opprimées) n'ont pas été perturbées par leur expérience sociale. En d'autres mots, selon le standpointisme, les femmes ont la capacité de se libérer des conséquences de l'expérience d'avoir été dominées (en revanche, il est impossible aux hommes de se libérer des conséquences de l'expérience d'avoir dominé). Un autre postulat discuté par Flax (1990) est celui qui affirme que la « réalité » possède une ou des structures que la raison et l'expérience peuvent découvrir. Elle remet en question le point de vue selon lequel il est possible que la raison et l'expérience permettent la « découverte» de ces « réalités ».

Même si on accepte que le vécu des femmes puisse constituer le point de départ de toute recherche sociologique, il faut encore préciser « de quelles 
femmes " ou encore, «à quel savoir de femme " les standpointistes se réfèrent. Le standpointisme est engagé à la pluralité, à la reconnaissance des différences entre les points de vue de femmes selon leur classe, leur race, leur culture. Il est possible de gérer ces dichotomies au niveau conceptuel et théorique, mais la réalité des femmes peut difficilement être organisée de façon duelle telle, par exemple, « hommes versus femmes ». Faut-il un ou des critères pour déterminer quelles seront les perspectives autres que classe sociale, race et culture, à partir desquelles nous cumulerons des connaissances (par exemple les victimes de viol, les femmes battues, les femmes avec enfants, les lesbiennes, les femmes n'ayant pas d'éducation, les femmes en milieu rural, etc.). De plus, il est difficile d'accepter que seules les expériences partagées par les femmes puissent orienter les sujets des recherches. Cela conduirait à ignorer certains événements importants, vécus à la fois par les hommes et par les femmes, et qui influencent la façon dont les femmes conçoivent le monde. Le défi pour les théoricien/ne/s, c'est de reconnaître la polysémie du standpointisme sans pour autant perdre l'essentiel de chaque point de vue. En raison de ce défi, certain/e/s ont critiqué le standpointisme, l'accusant de tendre vers le relativisme. Harding adresse cette critique en distinguant le relativisme « historique » et celui du « jugement ». Elle suggère que « a respect for historical relativism (or sociological or cultural) is always useful in starting one's thinking » (Harding, 1991, p. 52). Ainsi, les groupes ont chacun leur point de départ, leurs discours et leurs expériences. Une autre critique du standpointisme met l'accent sur la diversité. En acceptant ces différentes perspectives, que reste-t-il du principe unifiant les féministes, c'est-à-dire l'expérience partagée des femmes ? (Di Stefano, 1990; Bohan, 1993). De son côté, Haraway (1990) critique le standpointisme pour son « essentialisme ", ce besoin d'adopter des hypothèses au sujet d'une femme universelle.

Le féminisme postmoderne se situe à l'opposé des autres épistémologies féministes en adoptant une approche antipositiviste, critique envers l'objectivité et la découverte. La théorie du féminisme postmoderne n'adhère pas non plus à une redéfinition de la notion de l'objectivité. Comme l'affirme Lyotard (1979/1984), les grands discours justificatifs ne sont plus crédibles dans un cadre postmodeme. En effet, le postmodemisme se dissocie des métanarrations et de la perspective humaniste selon laquelle les individus sont conscients de leur existence à travers l'éducation, la rationalité et l'action (Lyotard, 1979/1984 ; Lather, 1991). La pensée postmoderne est sceptique face à toute déclaration de « vérité » et de « réalité » (Allen et Barber, 1992). De plus, elle défie l'attirance vers l'absolu et la certitude des connaissances. Elle ébranle aussi le contrôle de la pensée positiviste en rapport avec les théories et les pratiques qu'on retrouve dans les sciences sociales (Lather, 1991). Cette épistémologie attire les féministes critiques envers 
l'empirisme féministe et le standpointisme, car ces deux épistémologies s'accrochent à la possibilité de découvrir « la vérité » et « la réalité » (Flax, 1990). Le féminisme postmoderne jette un défi à la relation intime entre la science et le pouvoir masculin. Pour cette raison, les féministes d'orientation postmoderne rejettent l'empirisme - même la version modifiée de l'empirisme féministe - car elles ont perdu confiance non seulement dans la possibilité que l'empirisme puisse arriver à une description juste de la réalité, mais de façon plus globale, dans la possibilité même qu'une description de la réalité sociale soit jamais possible. Le féminisme postmoderne se tourne plutôt vers un engagement à l'égard de la pluralité, des efforts de déconstruction des croyances, de la vérité, du pouvoir, des relations entre les genres, de la création du genre (Allen et Barber, 1992) et s'éloigne de la "découverte ». Ces outils « interpretive insights and methods of critique " (Allan et Barber, 1992, p. 2) sont intéressants pour le développement de théories féministes.

Les critiques du féminisme postmoderne sont généralement centrées sur la « relativité » de cette approche (Hartsock, 1987a ; Harding, 1990).

[...] taken to its postmodernist extreme, every women's experience becomes the basis for a feminist epistemology, thereby deconstructing their experiences in the world to such an extent that feminists are in danger of erasing the solidarity that is needed in working towards women's liberation (Allen et Barber, 1992, p. 6).

Le problème semble se trouver entre les objectifs féministes et l'accent mis sur la déconstruction, l'analyse et le manque d'intérêt pour la "gênéralisation » afin d'arriver à une meilleure compréhension des femmes et de leur place dans le monde. Di Stefano (1990) insiste fortement pour que le féminisme ne soit pas situé dans la pensée postmoderne car, soutient-elle, c'est dans l'ère moderne que le rôle et l'importance du "genre " ont été fondés. Elle critique aussi le « postmoderne traditionnel ${ }^{7}$ » pour ne pas s'être préoccupé de ce concept de base. Selon Di Stefano, étant donné que le postmoderne découle de la "pensée ou de la philosophie mâle ", cette approche ne peut qu'exprimer les besoins des hommes. Toutefois, la même critique peut être faite envers l'empirisme féministe et le standpointisme. Dans ces deux cas, l'empirisme traditionnel et le marxisme ont provoqué, respectivement, l'élaboration de l'empirisme féministe et du standpointisme. La pensée féministe postmoderne peut être perçue comme une création

7. Bien que l'expression « postmoderne traditionnel » puisse représenter une contradiction, ici, nous l'utilisons simplement pour différencier le « postmoderne " du « féminisme postmoderne" 
générée par l'insatisfaction à l'égard du « postmodernisme mâle ». Les chercheuses qui adhèrent au féminisme empirique estiment que rejeter complètement l'empirisme équivaut à « jeter le bébé avec l'eau du bain » (Ussher, 1990). Parallèlement, le même raisonnement peut être appliqué à la pensée postmodeme et aux outils que cette dernière orientation offre pour la poursuite de l'agenda féministe. Mais il y a résistance, et certain/ne/s ont même suggéré que le féminisme postmoderniste représentait la demière ruse du patriarcat, peut-être son dernier effort pour minimiser les dégâts et empêcher son propre déclin (Morris, 1988).

Tableau 1

Une appréciation des épistémologies selon les objectifs féministes

\begin{tabular}{|c|c|c|c|c|}
\hline & $\begin{array}{l}\text { Empirisme } \\
\text { traditionnel }\end{array}$ & $\begin{array}{l}\text { Empirisme } \\
\text { féminisme }\end{array}$ & Standpointisme & $\begin{array}{l}\text { Féminisme } \\
\text { postmoderne }\end{array}$ \\
\hline Postulats & $\begin{array}{l}\text { siècle des lumières } \\
\text { pensée positiviste } \\
\text { objectivité } \\
\text { découverte }\end{array}$ & $\begin{array}{l}\text { siècle des lumières } \\
\text { pensée positiviste } \\
\text { objectivité (révisée) } \\
\text { découverte }\end{array}$ & $\begin{array}{l}\text { siècle des lumières } \\
\text { marxisme } \\
\text { pensée positiviste } \\
\text { objectivité (révisée) } \\
\text { découverte } \\
\text { fausse conscience }\end{array}$ & $\begin{array}{l}\text { anti (post) siècle } \\
\text { des lumières } \\
\text { anti (post) - } \\
\text { positivisme }\end{array}$ \\
\hline $\begin{array}{l}\text { Préjugés } \\
\text { reconnus }\end{array}$ & aucun & $\begin{array}{l}\text { objectifs féministes } \\
\text { genre } \\
\text { social (implicite) } \\
\text { historique } \\
\text { (implicite) }\end{array}$ & $\begin{array}{l}\text { objectifs féministes } \\
\text { genre } \\
\text { social (explicite) } \\
\text { historique } \\
\text { (implicite) }\end{array}$ & $\begin{array}{l}\text { objectifs férninistes } \\
\text { genre } \\
\text { social (explicite) } \\
\text { historique (implicite) }\end{array}$ \\
\hline $\begin{array}{l}\text { Point de } \\
\text { départ }\end{array}$ & neutre & intérêt des femrnes & $\begin{array}{l}\text { perspective de } \\
\text { groupes de femmes } \\
\text { spécifiques }\end{array}$ & $\begin{array}{l}\text { femme individuelle } \\
\text { pluralité }\end{array}$ \\
\hline Processus & $\begin{array}{l}\text { tests d'hypothèses } \\
\text { observation par les } \\
\text { sens }\end{array}$ & $\begin{array}{l}\text { tests d'bypothèses } \\
\text { observation par les } \\
\text { sens }\end{array}$ & $\begin{array}{l}\text { raison } \\
\text { expérience }\end{array}$ & $\begin{array}{l}\text { déconstruction } \\
\text { analyse } \\
\text { individuation } \\
\text { réduction }\end{array}$ \\
\hline résultats & $\begin{array}{l}\text { vérité(s) } \\
\text { généralisations } \\
\text { théories } \\
\text { métathéories } \\
\end{array}$ & $\begin{array}{l}\text { vérité(s) } \\
\text { généralisations } \\
\text { théories } \\
\text { métathéories }\end{array}$ & $\begin{array}{l}\text { vérité(s) } \\
\text { généralisations } \\
\text { théories } \\
\text { métathéories }\end{array}$ & $\begin{array}{l}\text { constructions } \\
\text { narratives }\end{array}$ \\
\hline risques & $\begin{array}{l}\text { fausse } \\
\text { représentation } \\
\text { « décontexuaa- } \\
\text { lisation " }\end{array}$ & $\begin{array}{l}\text { fausse } \\
\text { représentation } \\
\text { « décontextua- } \\
\text { lisation » }\end{array}$ & $\begin{array}{l}\text { fausse } \\
\text { représentation } \\
\text { relativisme }\end{array}$ & relativisme \\
\hline bénéfices & & conservatisme & nouvelles visions & nouvelles visions \\
\hline
\end{tabular}

Pour conclure cette section, quatre pensées épistémologiques - l'empirisme traditionnel, l'empirisme féministe, le standpointisme et le féminisme 
postmoderne - $s$ 'offrent à nous pour guider les recherches et redéfinir les connaissances en sciences sociales, et plus particulièrement en criminologie. Cependant, à partir de la discussion qui précède, l'empirisme traditionnel ne peut être retenu comme épistémologie habilitée à prendre en compte les besoins des féministes dans la poursuite de leurs objectifs politiques. Il reste donc trois alternatives. Le tableau 1 fait le résumé de ces épistémologies en incluant l'empirisme traditionnel en guise de comparaison.

\section{DÉFINIR LA RECHERCHE FÉMINISTE}

Il semble exister une conceptualisation de ce que la recherche féministe « devrait » accomplir et « comment » elle devrait le faire. Cependant, il est impossible de parler de méthodologie sans prendre en considération l'épistémologie du/de la chercheur/euse. En effet, l'étude des méthodologies de recherche ne peut se faire sans examiner plusieurs autres facettes telles que son agenda politique, son éthique, et son orientation épistémologique (Thompson, 1992 ; Cook et Fonow, 1990).

Selon Westkott (1979), les méthodes de recherche féministes doivent refléter l'expérience des femmes, et devraient être fondées sur les aspects concrets plutôt qu'abstraits de cette expérience. Pour Lather (1991), la recherche féministe doit découler des expériences, des désirs et des besoins des personnes opprimées. Malgré l'accent que Harding (1991) met sur les "explications scientifiques", le projet fondamental de la recherche féministe vise à améliorer les conditions de vie des femmes.

Examinons maintenant quelques études dans le domaine de la violence familiale afin de déterminer l'impact que peut avoir l'épistémologie du/de la chercheur/euse par rapport à la méthode de recherche, aux résultats obtenus, à leurs présentations et leurs interprétations.

\section{L'empirisme traditionnel}

En 1980, Straus, Gelles, et Steinmetz ont publié un livre intitulé Behind closed doors: Violence in the American family. Dans ce livre, les auteurs présentent les résultats d'une étude imposante sur la prévalence de la violence entre membres familiaux. Une partie importante des données furent recueillies en utilisant une échelle de violence - Conflict Tactics Scale (CTS) - qui situe la recherche dans le contexte de « conflits de famille " (Gelles, 1987). En appliquant les principes de la pensée positiviste, de l'empirisme traditionnel, de la notion traditionnelle d'objectivité et de "découverte", Straus et ses collègues ont pu démontrer non seulement que l'existence de la violence contre les conjointes était courante, mais aussi, que la violence contre les conjoints par les conjointes représentait un problème social de « nature " 
et d' « importance » comparable. De plus, ils ont constaté que les conjointes employaient même certaines formes de violence plus souvent que les conjoints.

Cette recherche est conforme aux conventions de la recherche empirique qui n'admet aucun biais ou préjugé et qui est dite « neutre » au point de départ. Les chercheurs expliquent « [...] the extent to which culture causes violence between family members [italique ajouté] (Straus et Hotaling, 1980; p. 5). Le choix du terme « culture » et de l'expression « membres familiaux " efface le contexte sexué. Les résultats représentent : 1) des données de sondage « fiables » et « valides », caractéristiques des plus importantes lorsqu'on décrit la « vérité », la « réalité », 2) des données à partir desquelles on peut généraliser, en raison de l'ampleur de l'échantillon étudié (le CTS fut distribué à 2143 familles américaines ; Straus, et al., 1980), et 3) de l'information pour une « $[. .$.$] special theory of violence... for the family group » (Straus$ et Hotaling ; 1980, p. 15). Comme plusieurs critiques féministes l'ont déjà mentionné, la méthode utilisée extrait le comportement de son contexte, ce qui mène à une fausse représentation de l'expérience des femmes abusées (Dobash et al., 1992 ; Kurz, 1993). L'étude pourrait être défendue en avançant qu'elle a défait le mythe selon lequel la « violence familiale » est rare. Toutefois, le coût associé à la fausse représentation de la réalité des femmes abusées et la création du « myth of sexual symmetry in marital violence " (Dobash et al., 1992) dépasse probablement les gains de cette étude.

\section{L'empirisme féministe}

L'étude de Rhodes (1992) montre comment l'empirisme féministe tente d'inclure l'expérience des femmes de façon plus ađéquate, tout en respectant les principes de l'empirisme traditionnel. Cette étude illustre aussi comment l'empirisme féministe se développe souvent « en réaction » à l'empirisme traditionnel. Rhodes développa une échelle pour mesurer « l'abus des conjoints » (spousal abuse). Par la suite, Rodenburg et Fantuzzo (1993) y réferent, l'appelant la Measure of Wife Abuse (MWA), et la suggérant comme grille pouvant remplacer la CTS. Selon ces chercheur/se/s, la MWA, en comparaison avec la CTS, permettrait de remettre dans le « contexte " l'abus fait aux femmes. De plus, cette nouvelle grille fut développée afin d'établir la fréquence et la sévérité de certaines formes d'abus non relevées par la CTS. Dans le cas de la CTS, les items furent développés à travers des discussions collégiales et informelles. En comparaison, les items de la MWA furent développés à partir : a) d'une analyse de contenu de 269 ordres de détention temporaires remis par les participantes d'un programme pour femmes abusées, b) de rapports de cas et $c$ ) de suggestions de directrices de maisons d'hébergement pour femmes victimes de violence conjugale 
(Rodenburg et Fantuzzo, 1993). Une préoccupation par rapport à la validité de l'échelle incita Rhodes à mener une série d'entrevues semi-structurées et approfondies auprès d'un certain nombre de femmes ayant été victimes d'abus. Elle dit avoir été « biaisée » en prenant pour acquis que les données obtenues en entrevues auraient moins tendance à sous-évaluer l'abus ${ }^{8}$. En adoptant ces stratégies de recherche, un outil empirique fut développé pour faire en sorte que les données obtenues puissent mieux refléter l'expérience des femmes. De plus, les résultats d'études qui suivront et qui emprunteront la MWA pourront rendre compte d'une plus grande variété de types d'abus car la MWA détaille un nombre plus élevé d'actes violents que la CTS (par exemple, actes d'abus sexuels et psychologiques) (Rodenburg et Fantuzzo, 1993). En d'autres mots, l'expérience et la réalité des femmes abusées peuvent être mieux représentées dans de futures études empiriques.

\section{Le standpointisme}

À titre d'exemples d'études menées dans le cadre du standpointisme, nous avons choisi la recherche-action de Mies (1984). Cet exemple ne doit pas mettre en équation la recherche-action et l'orientation épistémologique du standpointisme. Nous voulons plutôt mettre l'accent sur la perspective d'un groupe particulier de femmes opprimées, c'est-à-dire des femmes victimes de violence conjugale. L'agenda politique - et sa perspective biaisée - est clairement nommé dans l'étude de Mies. Comme le dit l'auteure, cette étude « [...] was not a systematic attempt to apply a certain methodology of social research [...] the main motive was to further the objectives of the action group " (p. 128). Comme pour Straus et ses collègues, déterminer l'étendue de la violence était l'élément principal de ce travail : pour le groupe de chercheuses" "Women Help Women», il était nécessaire de démontrer la prédominance de la violence contre les femmes dans le contexte familial afin d'obtenir des fonds pour ouvrir un maison d'hébergement pour femmes violentées par leur conjoint. Leur choix de méthode était basé sur le postulat théorique qui suggère que les membres ayant le moins de pouvoir dans la société ont accès à une vision de la réalité sociale plus complète que d'autres, précisément en raison de leur position désavantagée (Nielsen, 1990, p. 10). Par conséquent, le groupe «Women Help Women" avait accès à la " vérité ", à la « réalité " (un postulat, bien sûr, qui découle de la pensée marxiste, et lié au positivisme) par rapport à cette violence, et

8. Les résultats allaient en sens inverse.

9. Malgré notre emploi de l'étiquette de « chercheuses", ces femmes, très probablement, s'identifiraient plutôt à l'étiquette de militantes. 
elles se sont mobilisées pour en démontrer l'existence. La méthode qu'elles ont adoptée était bien différente des méthodes utilisées jusqu'à présent. Par exemple, elles ont organisé une manifestation où toutes les personnes ayant été témoins de cette violence étaient invitées à participer. Une autre stratégie fut de rendre compte, en détails, de l'expérience de femmes ayant été victimes de violence conjugale, afin d'analyser et de politiser cette problématique, et de générer une approche théorique s'y rapportant. Comme le suggère Henderson (1995), «Emancipatory inquiries are ideologies that seek to understand oppression in society and, through this understanding, transform it [...] The goal of emancipatory inquiry is social change » (p. 59). Un des résultats de cette recherche fut de changer la perception qu'avait le public au sujet de la prédominance de la violence contre les femmes dans le contexte familial. Plutôt que de considerrer cette violence comme rare, on la voit maintenant comme un problème de taille, sorti du domaine privé et présent dans le domaine public.

\section{Le féminisme postmoderne}

L'approche postmoderne ne confere pas une signification définitive et essentielle à l'expérience des femmes (Weedon, 1987). Dans ce cadre épistémologique, l'accent est mis sur l'utilisation du langage pour décrire les experiences. En nous servant de ce langage, nous donnons un sens à nos expériences en les nommant, en les racontant. La recherche de Gavey (1989) " seeks to locate women's accounts of their experiences of heterosexual coercion in relation to specific discourses concerning sexuality and sexual violence in particular, and interpersonal relationships and gender relations more generally » (p. 468). Comme elle le note, les discours principaux sur l'hétérosexualité amènent à percevoir le comportement sexuel coercitif mâle comme naturel. Ces discours soutiennent et perpétuent les relations de pouvoir déjà existantes. Ils tentent de faire autorité en cette matière en s'adressant au « sens commun » des gens. À travers cette stratégie, la plupart des individus construisent, et, par conséquent, reconnaissent leur réalité subjective dans ces discours (Gavey, 1989). Pour illustrer ce processus, Gavey examina deux discours dominants dans ses entrevues aupres de femmes : la « morale commode » et le discours du « besoin sexuel mâle ». En analysant la transcription d'une des entrevues, Gavey démontre l'adhésion à ces deux discours (par exemple, « it didn't mean that much to me ", «I seemed to have a lot of sex at the time ", « his manhood was going to be bashed if he didn't go to bed with me », « he was sort of desperate to have sex » p. 470). Tout en adhérant à la construction sociale de la sexualité mâle, l'expérience de coercition a tout de même laissé cette femme dans un état de « vide » et de dépression. Gavey suggère que ces émotions appartiennent à d'autres 
discours moins « hégémoniques », sur le droit d'une femme à avoir une sexualité satisfaisante, ou encore qu'elles sont liées à d'autres discours féministes sur l'exploitation de la femme dans les relations hétérosexuelles. Plutôt que d'insister sur la primauté d'une seule explication, une analyse postmoderne permet la coexistence d'interprétations qui, parfois, se contredisent. Cette recherche de « sens » dans ces différentes interprétations permet une richesse de points de vue et peut changer notre compréhension et notre construction de la réalité (Gavey, 1989). De cette manière, le féminisme postmoderne offre de nouvelles façons de travailler pour les féministes dans de nombreux domaines, y compris celui de la criminologie. Toutefois, la perspective postmoderne met en cause la représentation du construit de la victime dans cette même discipline. Dans le contexte légal actuel, cette possibilité place peut-être la victime dans une position encore plus dangereuse. Cette analyse nous met face à la dimension du consentement et à sa construction sociale. À cause de ses aspects complexes et peu familiers, et de son «penchant " pour le relativisme, le féminisme postmoderne risque d'aliéner plusieurs chercheur/euse/s (Di Stefano, 1990 ; Gavey, 1989).

\section{CONCLUSION}

Malgré les différentes approches disponibles pour mener la recherche féministe, il existe un consensus au niveau de ses objectifs. Il se résume dans l'engagement consistant à éliminer le patriarcat. À travers les exemples explorés, cet engagement se traduit par des efforts pour mieux faire entendre et comprendre la victime, lui faire une place centrale et concrète à travers les discours non seulement idéologiques et théoriques, mais aussi bien pratiques. Étant donné que la recherche féministe en est encore à ses débuts, il n'est pas évident de concevoir comment ces différentes traditions épistémologiques vont se conjuguer pour fournir une base de connaissances. La tolérance et l'acceptation à travers la communauté académique féministe et, on le souhaite, la communauté des chercheur/euse/s dans les domaines de recherche en sciences humaines et sociales, sauront encourager ce processus féministe qui, comme l'a affirmé Bertrand (1993), peut contribuer à la criminologie et à d'autres domaines (Landrine, 1995).

Chaque épistémologie féministe qui encadre le processus de recherche a quelque chose à apporter. La force de l'empirisme féministe se trouve dans son approche conservatrice à la recherche des connaissances. Le standpointisme et le féminisme postmoderne offrent plutôt l'innovation pour forger un savoir social. Chaque approche recèle un potentiel pour de nouvelles visions, de nouvelles perspectives, un nouveau savoir. 
De façon historique, les seules traditions épistémologiques furent celles des religions ${ }^{10}$ et de l'empirisme traditionnel pour la quête de connaissances. Présentement, des alternatives féministes émergent ; elles sont complexes, souvent à l'opposé l'une de l'autre, et parfois complémentaires. Basées sur différents postulats, elles partagent jusqu'à un certain point une base politique féministe. En ce moment, ces nouvelles épistémologies, et les cadres de recherche qui en découlent, sont suffisamment bien articulées pour tolérer la critique, la simplification et la comparaison. Non seulement il est nécessaire de pouvoir identifier les aspects qu'elles ont en commun, mais il est important d'arriver à questionner les contraintes que chacune impose au processus de recherche. Quelle que soit la perspective, à travers les détails et l'aspect global, l'intérêt est maintenant de voir comment se « tresseront ensemble $»$ les résultats de ces recherches.

\section{BIBLIOGRAPHIE}

ALLEN, K. R. et BARBER, K. M. (1992), « Ethical and epistemological tensions in applying postmodern perspective to feminist research $»$, Psychology of Women Quarterly, 16, 115.

BERTRAND, M.-A. (aout, 1993), « Feminist criticism : What it has done for theory and methodology in general and what it could do for Criminology in particular ", International Congress of Criminology, Plenary on theories and methodologies on criminality and criminalization, Conflicts and convergences, Budapest.

BERTRAND, M.-A. (1994), « Le pouvoir des théories féministes dans la reconsidération radicale des théries du contrôle social ", Théoretical Discourse/Discours théoriques, AEC, Montréal, 49-74.

BOHAN, J. S. (1993), « Regarding gender : Essentialism, constructionism, and feminist psychology ", Psychology of Women Quarterly, 17, 5-21.

COOK, J. A. et FONOW, M. M. (1990), «Knowledge and wornen's interests : Issues of epistemology and methodology in feminist sociological research », in J. McCarl Nielson (éd.), Feminist Research Methods : Exemplary Readings in the Social Sciences, Boulder, Westview Press, 69-93.

CRAWFORD, M. et MARECEK, J. (1989), « Feminist theory, feminist psychology : A bibliography of epistemology, critical analysis, and applications », Psychology of Women Quarterly, 13, 477-491.

DI STEFANO, C. (1990), «Dilemmas of difference : Feminism, modernity, and postmodernism », in L. J. Nicholson (éd.), Feminism/Postmodernism, New York : Routledge, 63-82.

10. Comme le montrent Lerner (1986) et plusieurs autres, les religions n'ont pas toujours été patriarcales, mais la force des femmes et des déesses reposait surtout dans la capacité de procréer. Toutefois, comme les prêtres des religions patriarcales, les prêtresses de ces religions précédentes étaient aussi consultées pour leurs connaissances divines, leur interprétation de symboles naturels, leurs prophéties, etc. 
DOBASH, R. P., DOBASH, R. E., WILSON, M. et DALY, M. (1992), " The myth of sexual symmetry in marital violence ", Social Problems, 39 (1), 71-91.

FLAX, J. (1990), « Postmodernism and gender relations in feminist theory ", in L. J. Nicholson (éd.), Feminism/Postmodernism, New York : Routledge, 39-62.

FOX KELLER, A. (1985), « Reflections on Gender and Science », New Haven, Connecticut, Yale University Press.

GAVEY, N. (1989), « Feminist poststructuralism and discourse analysis : Contributions to feminist psychology ", Psychology of Women Quarterly, 13, 459-475.

GELLES, R. G. (1987), "Family Violence », London, Sage Publications.

HARAWAY, D. (1990), «A Manifesto for Cyborgs : Science, Technology, and Socialist Feminism in the 1980s », in L. Nicholson (Ed.), Feminism/Postmodernism, New York, Routledge, 190-233.

HARDING, S. (1987), « Feminism and Methodology », Bloomington : Indiana University Press.

HARDING, S. (1989), «Is There a Feminist Method ?", In N. Tuana (ed.), Feminism and Science, Indiana, University Press.

HARDING, S. (1990), "Feminism, science, and the anti-Enlightenment critiques ", in L. Nicholson (éd.), Feminism/Postmodernism, New York, Routledge, 83-106.

HARDING, S. (1991), "Whose Science? Whose Knowledge? Thinking from Women's Lives », Ithaca, New York, Cornell University Press.

HARTSOCK, N. (1987a), « The feminist standpoint : Developing the ground for a specifically feminist historical materialism ", in S. Harding (éd.), Feminism and Methodology: Social Science Issues, Indiana, University Press.

HARTSOCK, N. (1987b), « Rethinking modernism : Minority vs. majority theories ", Cultural Critique, 7, 187-206.

HENDERSON, D. J. (1995), « Consciousness raising in participatory research : Method and methodology for emancipatory nursing inquiry ", Advances in Nursing Science, 17 (3), 58-69.

KREMER, B. (1990), «Learning to say no : Keeping feminist research for ourselves », Women Studies International Forum, 13, 463-467.

KURZ, D. (1993), « Physical assault by husbands : A major social problem », in R. J. Gelles Loseke (éds.), Current controversies on family violence, London, Sage Publications, 88-104.

LANDRINE, H. (1995), « Bringing Cultural Diversity to Feminist Psychology: Theory, research, and Practice ", Washington, D.C., American Psycholugical Association.

LANDRINE, H., KLONOFF, E. et BROWN-COLLINS, A. (1992), « Cultural diversity and methodology in feminist psychology : Critique, proposal, empirical example », Psychology of Women Quarterly, 16, 145-163.

LATHER, P. (1991), "Getting smart: Feminist Research and Pedagogy with/in the Postmodern ", New York, Routledge.

LERNER, G. (1986), "The Creation of Patriarchy », New York, Oxford University Press.

LYOTARD, J.-F. (1984), "The Post-Modern Condition : A Report on Knowledge», (G. Bennington et B. Massumi, traduction), Minneapolis : University of Minnesota Press (original publiê en 1979).

MIES, M. (1984), « Towards a methodology for feminist research », in E. Altbach, J. Clausen, D. Schultz, et N. Stephen (éds.), German Feminism : Readings in Politics and Literature, Albany, New York, State University of New York, 117-139. 
MORRIS, M. (1988), « The Pirate's Fiancee : Feminism, Reading, Postmodernism », London : Verso.

MURA, R. (1991), "Searching for subjectivity in the world of Sciences : Feminist viewpoints », The CRIAW paper series, Ottawa, CA : Institut Canadien de Recherche sur les Femmes.

NEILSEN, J. (1990), "Introduction », in J. Neilsen (ed.), Feminist Research Methods : Exemplary Readings in the Social Sciences, Boulder, Westview Press.

REINHARZ, S. (1992), « Feminist methods in social research », New York, Oxford University Press.

RHODES, N. R. (1992), « The Assessment of Spousal abuse : An alternative to the Conflict Tactics Scale », in E. C. Viano (éd.), Intimate Violence Interdisciplinary Perspectives, Washington, Hemisphere Publishing Corporation.

RODENBURG, F. A. et FANTUZZO, J. W. (1993), « The measure of wife abuse : Steps toward the development of a comprehensive assessment technique ", Journal of Family Violence, 8 (3), 203-228.

RORTY, R. (1979), "Philosophy and the Mirror of Nature ", Princeton : University Press.

SMITH, D. E. (1987), «The everyday world as problematic : A feminist sociology », Toronto, University of Toronto press.

STRAUS, M. A. et HOTALING, G. T. (1980), "The Social Causes of Husband-Wife Violence ", Minneapolis : University of Minnesota Press.

STRAUS, M.A., GELLES, R. et STEINMETZ, S. (1980), « Behind Closed Doors : Violence in the American Family », New York, Doubleday/Anchor.

TAVRIS, C. (1992), «The Mismeasure of Women », New York, Simon \& Shuster.

THOMPSON, L. (1992), « Feminist methodology for family studies », Journal of Marriage and the Family, 54 (fév.), 3-18.

USSHER, J. (1990), «Choosing psychology or not throwing the baby out with the bathwater ", in E. Burman (éd.), Feminist and Psychological Practice, London, Sage, 47-61.

WEEDON, C. (1987), « Feminist Practice and Poststructuralist Theory », Oxford, Blackwell.

WEISSTEIN, N. (1972), « Psychology construct the fernale; or the fantasy life of the male psychologist ", In M. H. Garshof (éd.), Roles women play : Readings toward women's liberation, Belmont, Californie, Brooks/Cole.

WESTKOTT, M. (1979), «Feminist criticism of the social sciences », Harvard Educational Review, 49 (4), 422-430. 